\title{
叶酸功能化介孔碳纳米球负载阿霉素的细胞靶向传递及可控释放
}

\author{
朱杰廖蕾朱丽娜继烈刘宝红* \\ (复旦大学化学系生物医学研究院 聚合物分子工程国家重点实验室 上海 200433)
}

\begin{abstract}
摘要 以粒径 $90 \mathrm{~nm}$ 的介孔碳纳米球作为靶向传药载体, 采用酸化处理改进了材料表面的亲疏水性及在溶液中的分散 性, 通过壳寡糖功能化, 并利用 EDC-NHS 将叶酸修饰到介孔碳纳米球表面. 通过共聚焦激光扫描显微镜及流式细胞仪 对实验体系的系统研究, 结果表明基于叶酸功能化的介孔碳纳米球能够有效提高负载药物对于 HeLa 细胞的跨膜转运 效率，叶酸阳性表达的 HeLa 细胞对于叶酸修饰的介孔碳纳米小球的吞噬效率明显高于叶酸阴性表达的 MCF-7 细胞. 对 $\mathrm{HeLa}$ 细胞毒性的定量分析表明叶酸的靶向作用在提高介孔碳纳米球内吞效率的同时，进一步提高了阿霉素对于 $\mathrm{HeLa}$ 细胞的毒性.
\end{abstract}

关键词 介孔; 纳米材料; 癌症; 靶向治疗

\section{Folate Functionalized Mesoporous Carbon Nanospheres as Nanocarrier for Targetted Delivery and Controlled Release of Doxorubicin to HeLa Cells}

\author{
Zhu, Jie Liao, Lei Zhu, Lina Kong, Jilie Liu, Baohong* \\ (Department of Chemistry, Institute of Biomedical Sciences and State Key Lab of Molecular Engineering of Polymers, \\ Fudan University, Shanghai 200433)
}

\begin{abstract}
Ordered mesoporous carbon is a kind of novel carrier for intracellular drug release. There are few reports on the use of mesoporous carbon nanospheres (MCNs) as the transmembrane deliverer in human cancer cells; on the other hand, the particle size of MCNs synthesized by hard templates is usually larger than $100 \mathrm{~nm}$. It is accepted that the optimal size of a transmembrane delivery vehicle should be less than $100 \mathrm{~nm}$ in diameter and the surface should be hydrophilic to circumvent clearance by macrophages, to maximize circulation times and targeting ability. In this work, MCNs with a diameter of $c a$. 90 $\mathrm{nm}$ have been developed as a targeted drug delivery system of an anticancer drug, doxorubicin (DOX). The small MCNs were synthesized using triblock copolymer Pluronic F127 as a template. The MCNs were first treated by acid to improve its dispersion property in an aqueous solution, and then modified by folic acid through EDC-NHS. The structure of the MCNs was well characterized by transmission electron microscopy, small-angle X-ray scattering, nitrogen adsorption/desorption and dynamic light scattering. pH-dependent drug release is successfully achieved due to the supramolecular $\pi-\pi$ stacking between DOX and the carbonaceous structures. By effective passive and active targeting, MCNs can be readily internalized into HeLa cells, where the carried DOX can be efficiently released in the acidic microenvironment of the tumors for further therapy. The results from confocal laser scanning microscope and flow cytometry demonstrated that the cellular uptake efficiency of MCNs toward HeLa cells was increased through the functionalization with folic acid, and the folate modified MCNs show much higher endocytosis properties toward HeLa cells (folate receptor positive) than toward MCF-7 cells (folate receptor negative). The cytotoxicities toward HeLa cells were studied by MTT method, which indicated that the cytotoxicities of DOX loaded mesoporous carbon nanoparticles was also enhanced due to the introduction of folic acid and targeted delivery, while the cytotoxicities of MCNs show very good biocompatibility toward both HeLa and KB cells.
\end{abstract}

Keywords mesoporous; nanoparticle; cancer; targeted therapy

\section{1 引言}

传统的化学治疗剂非特异性地分散到人体中，其毒 性在作用于癌症细胞的同时, 也会影响到人体的正常细 胞 ${ }^{[1]}$, 因而我们需要发展一类新型的药物载体来解决这 一问题. 药物载体的开发面临的最大挑战是如何能在传
递足够药量到癌症组织的同时减少对其他组织的副作 用 ${ }^{[2]}$. 如今很多纳米载体已经被用于提高抗癌药物的临 床价值, 如脂质体 ${ }^{[3]}$ 、碳纳米管 ${ }^{[4]}$ 、介孔硅材料等 ${ }^{[5]}$. 其 中介孔材料具有非常独特的结构, 其比表面积高、孔容 大、介孔结构有序的特性有助于提高药物负载量 ${ }^{[5]}$, 并 且纳米尺度的介孔材料能高效地携带药物穿过细胞膜,

\footnotetext{
*E-mail: bhliu@fudan.edu.cn

Received September 18, 2012; published December 20, 2012.

Supporting information for this article is available free of charge via the Internet at http://sioc-journal.cn

Project supported by the National Natural Science Foundation of China (No. 20925517) and the State Key Lab of Electroanalytical Chemistry of Changchun Institute of Applied Chemistry (No. SKLEAC201101).

项目受国家自然科学基金(No. 20925517)和长春应用化学研究所电分析化学国家重点实验室(No. SKLEAC201101)资助.
} 
将抗癌药物在癌细胞内有效释放, 因而介孔材料是一种 十分理想的药物载体, 这类材料已经被广泛应用到生物 科技和纳米医药中. 介孔碳材料是继介孔硅材料之后又 一种新型的药物载体, 它们具体比表面积大、孔容高、 药物负载量高等特点, 其可控的介孔结构可以保护目标 药物, 防止其在到达癌症组织前过早释放. 目前介孔碳 纳米材料作为癌细胞跨膜载体的报道还较少, 并且大都 采用硬模板法制备, 得到的粒径在 $100 \mathrm{~nm}$ 以上 ${ }^{[6]}$. 然而, 理想的跨膜载体其粒径应该在 $100 \mathrm{~nm}$ 以下, 并且具有 亲水的表面, 从而可以延长体内循环时间, 增强到达目 标组织的能力 ${ }^{[7]}$.

我们在前期的工作中首次利用粒径 $90 \mathrm{~nm}$ 左右的介 孔碳纳米小球(Mesoporous Carbon Materials, MCNs)作 为为抗癌药物阿霉素(Doxorubicin, $\mathrm{DOX}$ )的载体, 通过 超分子相互作用实现了 $\mathrm{pH}$ 可控的药物释放, 即在与人 体血液及正常组织相近的近中性环境中, DOX 可以稳定 地负载于介孔碳中, 而在癌症组织的弱酸性环境中快速 释放, 这一特性对降低 DOX 的副作用起到了非常积极 的作用 ${ }^{[8]}$. 然而 $\pi-\pi$ 堆积这一超分子相互作用并不能适 用于所有药物, 为了区分正常细胞和癌细胞, 靶向能力 对于纳米碳材料的进一步发展至关重要. 在本文中我们 通过酸化处理的方式首先改进了 $\mathrm{MCNs}$ 的亲水性, 通过 壳寡糖的功能化, 采用 1-乙基-3-(3-二甲基氨丙基)-碳 化二亚胺(EDC)和 $N$-羟基琥珀酰亚胺(NHS) 将叶酸 (Folate Acid, FA)修饰到 MCNs 表面(FA-MCNs), 起到靶 向作用. 利用共聚焦激光扫描显微镜(Confocal Laser Scanning Microscope, CLSM)和流式细胞仪(Flow Cytometry, FCM)对该系统进行了比较研究, 我们发现叶酸
功能化有效地提高了 MCNs 对于 HeLa 细胞的跨膜转运 效率, 并且叶酸阳性表达的 HeLa 细胞对于 FA-MCNs 的吞噬效率明显高于叶酸阴性表达的 MCF-7 细胞. 通 过对于 HeLa 细胞毒性的定量表征，我们发现这一靶向 作用使得具有更高内吞效率的 FA-MCNs 相比于 MCNs 能更好地发挥药物的毒性.

\section{2 结果与讨论}

\section{1 介孔碳纳米材料的合成及功能化}

我们以三嵌段共聚物 Pluronic F127 作为模板, 采用 在低浓度下水热合成的方法得到了具有纳米结构及均 一粒径的介孔碳纳米球 $(\mathrm{MCNs})^{[9]}$. 图 1a 为 $\mathrm{MCNs}$ 的透 射电子显微镜(Transmission Electron Microscopy, TEM) 照片, 通过量取其中 100 个 MCNs 的直径, 我们可以得 到其粒径分布(图 1b), 该纳米材料的平均直径在 $87 \mathrm{~nm}$ 左右. 小角 $\mathrm{X}$ 光散射 (Small-Angle X-ray Scattering, SAXS) 图在横坐标 $q$ 等于 0.73 和 $1.27 \mathrm{~nm}^{-1}$ 处得到两个 散射峰(图 1c), 两峰处的 $q$ 值的比为 $1: 3^{1 / 2}$, 这两个峰 可以被归属为面心立方 $\operatorname{Im} 3 m$ 结构的 110 面和 211 面. MCNs 的比表面积为 $746 \mathrm{~m}^{2} \cdot \mathrm{g}^{-1}$, 孔容为 $0.76 \mathrm{~cm}^{3} \cdot \mathrm{g}^{-1}$, 其孔径分布在约 $2.7 \mathrm{~nm}$ (图 1d), 良好的介孔结构使其成 为潜在的药物载体.

为了改善 $\mathrm{MCNs}$ 表面的亲疏水性和在溶液中的分 散性, 我们采用酸化(硝酸硫酸混合体系)处理的方式, 成功在其表面引入羧基. 酸化处理后的 MCNs 经动态光 散射(Dynamic Light Scattering, DLS)表征, 得到其表面 电位为 $-29.6 \mathrm{mV}$. 为了进一步进行叶酸的功能化, 我
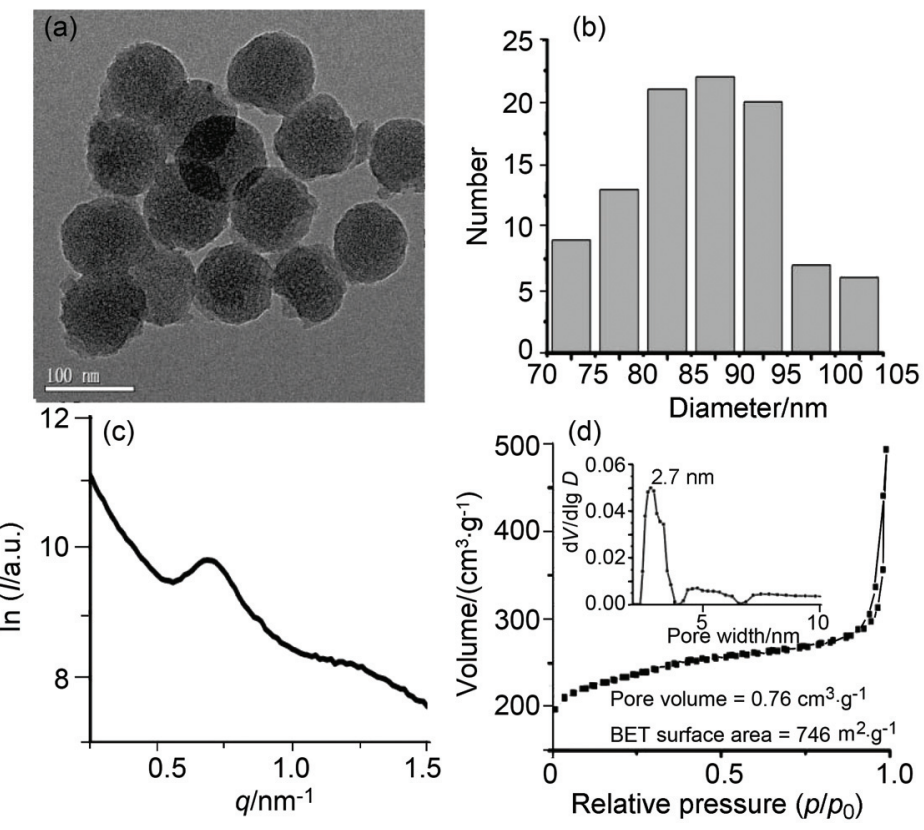

图 1 介孔碳纳米小球的材料表征图

Figure 1 The characterizations for the MCNs

(a) The TEM image of MCNs; (b) Histogram showing the size distribution of MCNs, 100 MCNs were measured from TEM images; (c) the SAXs pattern of MCNs; (d) the nitrogen adsorption/desorption isotherms of MCNs 

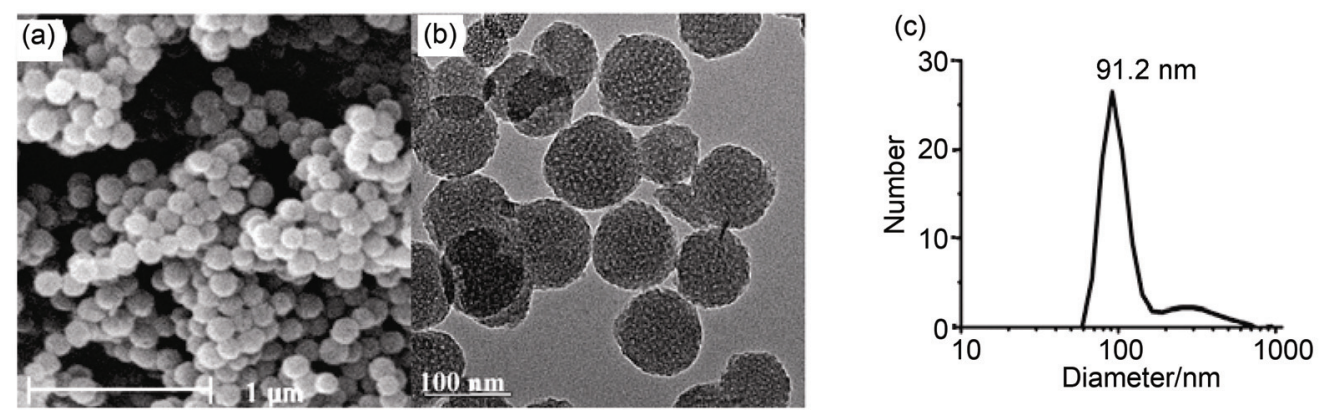

图 2 叶酸修饰的介孔碳纳米小球的材料表征图

Figure 2 The characterizations for the FA-MCNs

(a) The SEM image of FA-MCNs; (b) the TEM image of FA-MCNs; (c) size distribution of FA-MCNs measured by a Nanosizer ZS-90 instrument

们在材料表面吸附了一层壳寡糖, 此时 MCNs 的表面电 位从 $-29.6 \mathrm{mV}$ 提高到了- $10.8 \mathrm{mV}$. 在相关文献中曾 有用多糖对碳纳米管进行功能化的报道, Dyson 等 ${ }^{[10]}$ 成 功地在多糖修饰的碳纳米管表面引入叶酸配体, 并进一 步利用 $\pi-\pi$ 堆积将抗癌药物阿需素负载到碳纳米管表 面, 实现了靶向传药的目的. 我们受此启发, 以 EDC-NHS 活化叶酸表面的羟基, 通过共价键将叶酸修 饰到了 MCNs 表面(FA-MCNs). 众所周知, 与叶酸结合 的分子探针及纳米颗粒已经被广泛用作对叶酸受体过 表达的细胞进行靶向传质, 例如 HeLa 和 KB 细胞 ${ }^{[11]}$. 经 叶酸修饰后的 MCNs (FA-MCNs)的扫描电子显微镜和 透射电子显微镜图见图 $2 \mathrm{a}$ 和图 $2 \mathrm{~b}$, 可以看到该纳米材 料在一系列的处理后依然保持了良好的形貌, DLS 结果 表明 FA-MCNs 的粒径在约 $91.2 \mathrm{~nm}$, 值得一提的是此时 粒径的测量是将 FA-MCNs 分散到了磷酸盐缓冲溶液 (Phosphate Buffer Solution, PBS)中, 可以看出修饰后的 FA-MCNs 表面已经显示出了良好的亲水性.

\subsection{FA-MCNs 对 HeLa 细胞的靶向传药}

DOX 首先被负载于 MCNs 和 FA-MCNs 上(DOX@ MCNs, DOX@FA-MCNs), MCNs 是一种具有缺陷的石 墨结构, 这样的结构使得它可以与碳纳米管等材料一样 与具有芳香性的有机分子产生 $\pi-\pi$ 相互作用, 从而实现 MCNs 对于 DOX 的负载 ${ }^{[8]}$. 在本工作中, 我们选取了 DOX 负载量接近的 $\mathrm{MCNs}$ (负载量 $27.4 \mathrm{wt} \%$ ) 及 FA-MCNs(负载量 $26.7 \mathrm{wt} \%$ ), 并选取叶酸受体阳性的 HeLa 细胞和叶酸受体阴性的 MCF-7 细胞进行了一系列 对比实验.

图 3 是 CLSM 结果, 其中蓝色信号来自于 4',6-二㜆 基-2-苯基吲哚(4',6-diamidino-2-phenylindole, DAPI), 接 收波长为 $420 \sim 460 \mathrm{~nm}$, 代表了染色后的细胞核信号; 红色信号来自于 DOX, 接收波长为 $650 \sim 750 \mathrm{~nm}$; 所有 的图片都在相同的仪器参数下拍摄, 材料浓度均为 50 $\mu \mathrm{g} / \mathrm{mL}$. 图 3a 是与 DOX@FA-MCNs 共培养 $0.5 \mathrm{~h}$ 后的 HeLa 细胞的共聚焦照片, 从图中我们可以清晰地看到 明亮的红色苂光信号充满了整个 HeLa 细胞, 包括胞浆 部分和与蓝色信号重叠的细胞核区. 图 $3 \mathrm{~b}$ 是与

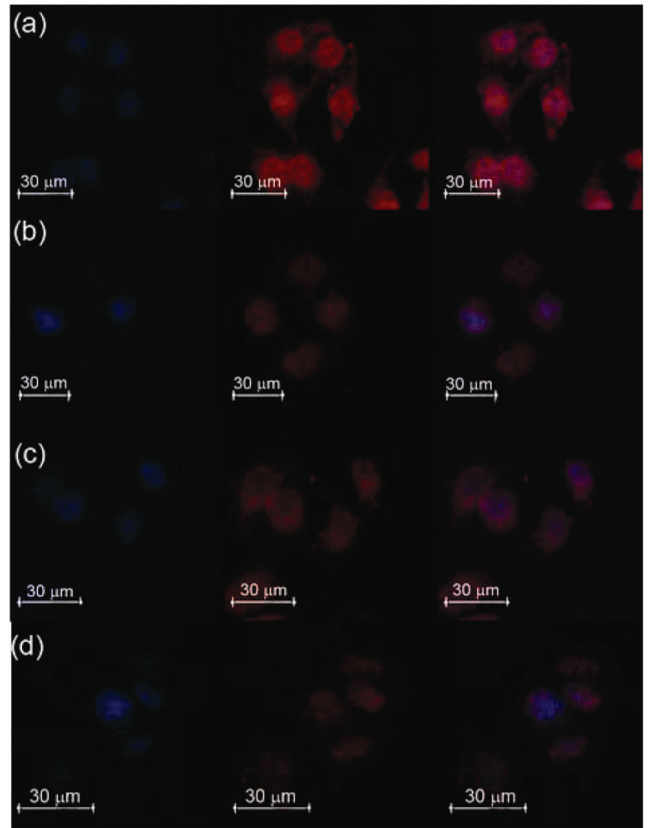

图 3 与 DOX@FA-MCNs (a), DOX@MCNs (b), 依次与 FA、 DOX@FA-MCNs (c)培养后的 HeLa 细胞的荧光共聚焦表征图及与 DOX@FA-MCNs 培养后的 MCF-7 的荧光共聚焦表征图(d)

Figure 3 The confocal microscopy of HeLa cells cultivated with DOX@FA-MCNs (a), DOX@MCNs (b), the confocal microscopy of FA blocked HeLa cells cultivated with DOX@FA-MCNs (c) and that of MCF-7 cells cultivated with DOX@FA-MCNs (d)

DOX@MCNs 共培养 $0.5 \mathrm{~h}$ 后的 HeLa 细胞的共聚焦照 片, 图 3c 是与叶酸预培养 $2 \mathrm{~h}$ 后, 再与 DOX@FAMCNs 共培养 $0.5 \mathrm{~h}$ 的 HeLa 细胞的共聚焦照片, 可以看 出由于叶酸受体与配体的相互作用被抑制, 两者的红色 荧光信号明显低于图 3a 中的红色荧光信号. 而图 3d 是 与 DOX@FA-MCNs 共培养 $0.5 \mathrm{~h}$ 后的 MCF-7 细胞的共 聚焦照片, 由于其表面叶酸受体的量少于 HeLa 细胞, 所以减弱了其与 FA-MCNs 之间的相互作用, 因而其红 色荧光信号也弱于图 3a 中的红色荧光信号. 同时我们 也对纯药物 DOX 进行了观察, 其结果见图 S1(支持信 息), 从结果我们可以看出无论有无材料作为载体, DOX 都主要存在于细胞的核区, DOX 的作用机理是通过干扰 
拓扑异构酶 II-DNA 来破坏 DNA 双链 ${ }^{[12]}$.

由于激光共聚焦苂光显微镜拍摄细胞的数量有限, 并不能作为荧光定量的依据. 为了更好地比较以上四种 实验条件下所得到的苂光强度, 我们进一步采用 FCM 对荧光强度进行了定量比较(见图 4), 每组实验我们都 测试了约 30000 个细胞的苂光强度, 实验结果表明与 DOX@FA-MCNs 共培养 $0.5 \mathrm{~h}$ 的 HeLa 细胞拥有最高的 苂光强度, 该结果与 CLSM 的结果完全一致.

值得一提的是, 我们前期研究结果表明 MCNs 本身 并不能进入细胞核 ${ }^{[8]}$, 这里细胞核内的红色荧光信号来 自于 DOX, 当 DOX@MCNs、DOX@FA-MCNs 进入细 胞后, 由于 $\mathrm{pH}$ 值变化使 $\mathrm{DOX}$ 加速释放, 从而进入了细 胞核. DOX 这种抗癌药的作用机理是通过干扰拓扑异 构酶 II-DNA 来破坏 DNA 双链, 其高毒性给病人带来 了极大的毒副作用 ${ }^{[12]}$, 而我们以介孔碳纳米材料为载 体实现 $\mathrm{pH}$ 可控的 DOX 释放, 即在人体弱碱性的生理环 境下释放缓慢, 而在癌细胞内酸性的环境中快速释放 (见图 5), 从而减少了其对人体正常组织的破坏 ${ }^{[8]}$; 同时 该纳米材料经FA修饰, 可以针对FA受体过表达的癌细 胞例如 HeLa 进行靶向治疗(主动靶向), 进一步提高了 其实用价值; 同时粒径在 $100 \mathrm{~nm}$ 以下的纳米材料也较 易在癌症组织的缺陷部位聚集, 起到了被动靶向的作 用 ${ }^{[7]}$.

\subsection{FA-MCNs 的细胞毒性比较}

通过 CLSM 和 FCM 的表征我们已经清楚地看到了 FA-MCNs 对 HeLa 细胞的靶向作用, 这为细胞毒性实验 打下了基础. 采用 3-(4,5-二甲基噻唑-2)-2,5-二苯基四氮 唑溴盐(MTT)法, 我们对 DOX@FA-MCNs, DOX@ MCNs 和 DOX 在不同培养时间(24, 48 h)、不同培养浓 度(50,500 $\mu \mathrm{g} / \mathrm{mL})$ 下的细胞毒性进行了定量比较(图 5). 图 5 显示 DOX@FA-MCNs 在低浓度 $(50 \mu \mathrm{g} / \mathrm{mL})$ 时, $24 \mathrm{~h}$ 的细胞存活率仅为 $43.7 \%, 48 \mathrm{~h}$ 的细胞存活率更是降到 了 $29.4 \%$, 而在高浓度 $(500 \mu \mathrm{g} / \mathrm{mL})$ 下, $24 \mathrm{~h}$ 的细胞存活 率为 $32.9 \%, 48 \mathrm{~h}$ 的细胞存活率进一步降低到 $15.9 \%$; 而 没有靶向作用的对照组 DOX@MCNs，在低浓度(50 $\mu \mathrm{g} / \mathrm{mL}$ ) 时的细胞存活率分别为 57.9\% (24 h) 和 55.8\% $(48 \mathrm{~h})$, 当浓度升高到 $500 \mu \mathrm{g} / \mathrm{mL}$ 时, 其存活率也只达 到了 $43.1 \%(24 \mathrm{~h})$ 和 $33.6 \%$ (48 h). 毒性实验的结果进一 步表明, 叶酸的靶向作用通过增强 MCNs 与 HeLa 细胞 的相互作用提高了细胞内吞效率, 而经过内吞作用进入 细胞的 DOX 在 $\mathrm{pH}$ 值的驱动下很好的得以释放, 从而自 行进入细胞核中, 最终破坏了 DNA 双链, 从而达到了 增强细胞毒性的目的. 同时图 5 的结果也表明 DOX 本 身的毒性高于DOX@FA-MCNs 和 DOX@MCNs. 同时, 我们也对材料的毒性进行了表征(图 S2, 支持信息), 结
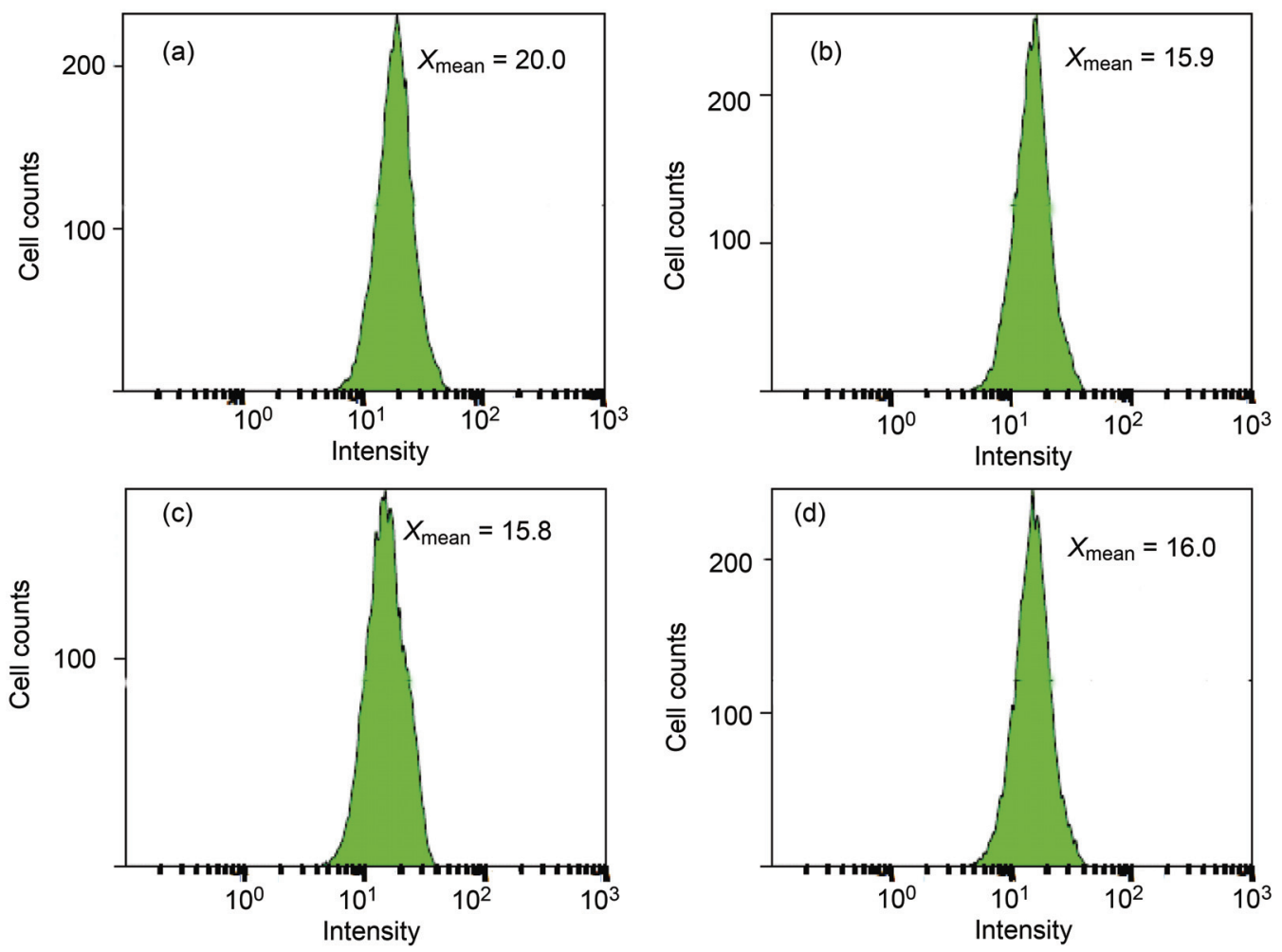

图 4 与 DOX@FA-MCNs (a), DOX@MCNs (b), 依次与 FA、DOX@FA-MCNs (c)培养后的 HeLa 细胞的流式细胞表征图及与 DOX@FA-MCNs 培养后的 MCF-7 的流式细胞表征图(d).

Figure 4 The flow cytometry analysis of HeLa cells cultivated with DOX@FA-MCNs (a),DOX@MCNs (b), the flow cytometry analysis of FA blocked HeLa cells cultivated with DOX@FA-MCNs (c) and that of MCF-7 cultivated with DOX@FA-MCNs (d) 


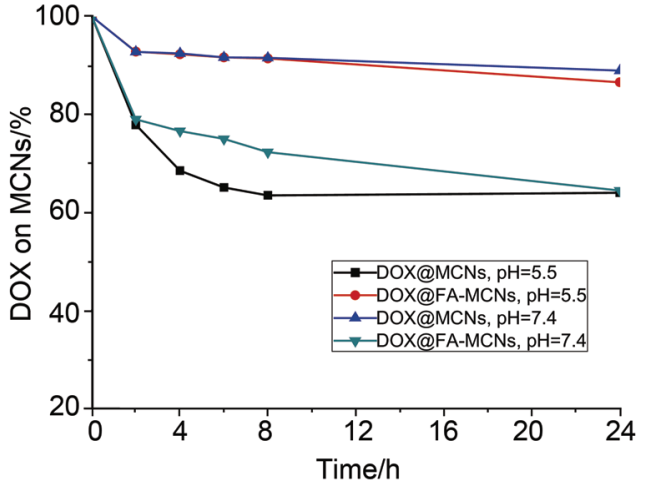

图 5 DOX@MCNs 及 DOX@FA-MCNs 在不同 $\mathrm{pH}$ 下的药物释放曲 线

Figure 5 pH-dependent drug release of DOX from $\mathrm{MCNs}$ and FA-MCNs with an initial concentration of $1 \mathrm{mg} / \mathrm{mL}$

果表明 MCNs 和 FA-MCNs 对 HeLa 细胞和 KB 细胞具 有较好的生物相容性.

\section{3 结论}

我们通过酸化处理的方式改善了介孔碳纳米球 $(\mathrm{MCNs})$ 表面的亲疏水性, 采用壳寡糖的功能化及 EDC-NHS 相互作用将叶酸FA 修饰到 MCNs 表面, 通过 对 CLSM 和 FCM 的系统比较和研究, 证明了叶酸的功 能化修饰能够提高 MCNs 对于 HeLa 细胞的跨膜转运效 率, 并且叶酸阳性表达的 HeLa 细胞对于FA-MCNs 的吞 噬效率明显高于叶酸阴性表达的 MCF-7 细胞. HeLa 细 胞毒性定量实验表明叶酸的靶向作用在提高 MCNs 内 吞效率的同时, 也使得 DOX@FA-MCNs 相较于 DOX@MCNs 具有更高的毒性.

\section{4 实验部分}

\section{1 实验药品}

三嵌段共聚物 Pluronic F127, EDC, NHS, FA 购自 Sigma-Aldrich, 甲醛、苯酚、多聚甲醛、氢氧化钠、硫 酸、硝酸购自上海化学品公司, 盐酸阿霉素(DOX)购自 上海华蓝化学品公司. 壳寡糖 $\left(M_{\mathrm{w}}: 30000 \sim 60000 \mathrm{Da}\right)$ 购 自合肥博美生物科技公司.

\subsection{MCNs 的合成和叶酸修饰}

本文中使用的碳球是按照之前的报道合成的, 简单 而言, $0.6 \mathrm{~g}$ 苯酚、 $2.1 \mathrm{~mL}$ 福尔马林 $(37 \mathrm{wt} \%$ ) 和 $15 \mathrm{~mL} 0.1$ $\mathrm{mol} / \mathrm{L}$ 的氢氧化钠溶液混合, 在 $70{ }^{\circ} \mathrm{C}$ 搅拌 $0.5 \mathrm{~h}$ 得到低 分子量的树脂. 之后加入 $0.96 \mathrm{~g}$ 三嵌段共聚物 Pluronic F127 的水溶液 $15 \mathrm{~mL}$. 体系在 $66{ }^{\circ} \mathrm{C}$ 以 $(340 \pm 40) \mathrm{r} / \mathrm{min}$ 的转速搅拌 $2 \mathrm{~h}$, 之后加入 $50 \mathrm{~mL}$ 水, 搅拌 $16 \sim 18 \mathrm{~h}$ 后, $17.7 \mathrm{~mL}$ 上述溶液被转移到水热釜中, 并用 $56 \mathrm{~mL}$ 水稀 释, 之后在 $130{ }^{\circ} \mathrm{C}$ 水热 $24 \mathrm{~h}$. 得到的 $\mathrm{MCNs}$ 用混合酸体 系(硫酸和硝酸的体积比是 3)超声处理 $4 \mathrm{~h}$, 酸处理后的 材料离心洗涤直到洗涤液呈中性.
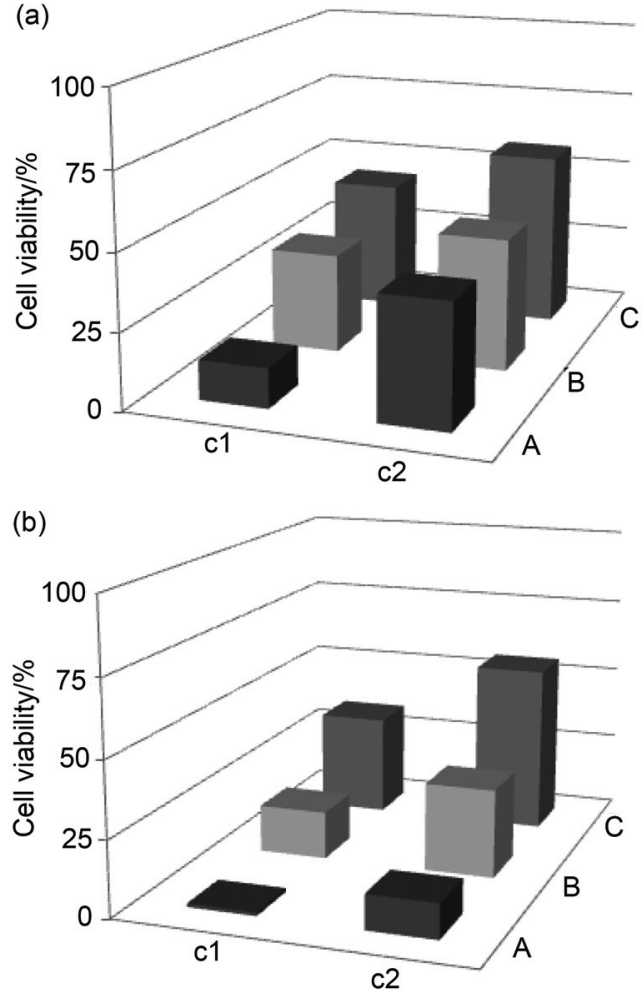

图 6 不同材料对 HeLa 细胞的毒性表征

Figure 6 The cytotoxicities of DOX loaded MCNs, FA-MCNs and DOX against HeLa cells

The cytotoxicities of DOX (A), DOX loaded FA-MCNs (B) and DOX loaded MCNs (C) against HeLa cells after $24 \mathrm{~h}$ (a) and after $48 \mathrm{~h}$ (b), c1 and $\mathrm{c} 2$ represent different concentrations. The details are shown in the supporting information

$40 \mathrm{mg}$ 壳寡糖溶解于 $0.1 \mathrm{~mol} / \mathrm{L}$ 氢氧化钠溶液中, 之 后加入 $20 \mathrm{mg}$ 酸化处理过的 MCNs, 超声 $20 \mathrm{~min}$ 后, 继 续摚拌 $16 \mathrm{~h}$ 后, 洗涤干燥, 得到壳寄糖修饰的 MCNs. 将上述材料 $20 \mathrm{mg}$ 超声分散于 $\mathrm{pH} 7.4$ 的缓冲溶液中, 之 后分别加入 $30 \mathrm{mg} \mathrm{FA}, 60 \mathrm{mg}$ EDC 和 $60 \mathrm{mg}$ NHS, 在 4 ${ }^{\circ} \mathrm{C}$ 下反应过夜, 得到 FA-MCNs.

\section{$4.3 \mathrm{DOX}$ 的负载}

$3 \mathrm{mg}$ DOX 与 $10 \mathrm{mg} \mathrm{MCNs} / \mathrm{FA}-\mathrm{MCNs}$ 加入到 $\mathrm{pH}=$ 7.4 的 PBS 缓冲溶液中摚拌 $24 \mathrm{~h}$, 之后用 $\mathrm{pH} 7.4$ 的 PBS 溶液透析 $2 \mathrm{~d}$ (每 $6 \mathrm{~h}$ 换一次水)去除未负载上去的 DOX. 所有的透析液都被收集起来用于紫外定量测试，从而得 到 DOX 在材料上的负载量.

\section{4 细胞成像实验}

HeLa 细胞用含有 $10 \%$ 血清(Fetal Bovine Serum)的 DMEM(Dulbecco's modified Eagle's medium)培养基在 $37{ }^{\circ} \mathrm{C}$ 及 $5 \% \mathrm{CO}_{2}$ 的条件下培养过夜 $\left(5 \times 10^{8} / \mathrm{L}\right.$ 细胞在 14 $\mathrm{mm}$ 的盖玻片上粘附生长). 培养 $24 \mathrm{~h}$ 后, 细胞用 PBS 溶 液洗涤 3 次, 之后根据不同的实验需要进行后续实验. 对于需要FA 预处理的对比组而言, 首先加入含 FA 0.5 $\mathrm{mg} / \mathrm{mL}$ 的 DMEM 溶液 $2 \mathrm{~mL}$ ，对于不需要 FA 预处理的 两个对比组, 则直接加入 DMEM 培养液 $2 \mathrm{~mL}$; 培养 $2 \mathrm{~h}$ 
后, 将上述溶液取出, 用 PBS 溶液洗涤 3 次, 之后在 FA 预处理的对比组中加入 $2 \mathrm{~mL}$ DOX@FA-MCNs (50 $\mu \mathrm{g} / \mathrm{mL}$ ). 在另两个对比组中, 分别加入 $2 \mathrm{~mL}$ DOX@FA-MCNs (50 $\mu \mathrm{g} / \mathrm{mL}$ ) 和 2 mL DOX@MCNs (50 $\mu \mathrm{g} / \mathrm{mL}$ ), 继续培养 $30 \mathrm{~min}$. 对于 MCF-7 细胞实验步骤大 致与 $\mathrm{HeLa}$ 细胞相同, 细胞用含有 $10 \%$ 血清(Fetal Bovine Serum)和 1\% 胰岛素的 MEM (modified Eagle's medium) 培养基在 $37{ }^{\circ} \mathrm{C}$ 及 $5 \% \mathrm{CO}_{2}$ 的条件下培养过夜 $\left(5 \times 10^{8} / \mathrm{L}\right.$ 细胞在 $14 \mathrm{~mm}$ 的盖玻片上粘附生长). 培养 $24 \mathrm{~h}$ 后, 细 胞用 PBS 溶液洗涤 3 次, 之后加入 MEM 培养液 $2 \mathrm{~mL}$, 培养 $2 \mathrm{~h}$ 后, 将上述溶液取出, 用 PBS 溶液洗涤 3 次, 之 后加入 2 mL DOX@FA-MCNs $(50 \mu \mathrm{g} / \mathrm{mL})$.

对于 CLSM 成像实验而言, 上述细胞培养后用 PBS 溶液洗涤 3 次, 加入浓度为 $4 \%$ 的多聚甲醛溶液, 细胞核 用 $5 \mu \mathrm{g} / \mathrm{mL}$ 的 DAPI(含 $10 \%$ 甘油) 染色. 之后用莱卡共聚 焦激光扫描显微镜(SP5)在 63 倍油镜下进行表征; DAPI 用 $405 \mathrm{~nm}$ 激光激发, 在 $425 \sim 460 \mathrm{~nm}$ 处接收; DOX 用 $488 \mathrm{~nm}$ 激发, 在 $550 \sim 650 \mathrm{~nm}$ 处接收.

对于流式细胞仪表征而言, 细胞以 $5 \times 10^{8} / \mathrm{L}$ 的浓度 培养在 $60 \mathrm{~mm}$ 的培养皿中 $\left(5 \times 10^{5}\right.$ 个), 之后的实验操作 如上, 在培养结束后, 上述细胞用胰酶消化, 并用培养 基及 PBS 溶液各洗涤一次, 最后用 EPICS ALTRA 自动 流式细胞仪(BECKMAN COULTER)表征. DOX 用 488 $\mathrm{nm}$ 激发, 在 $550 \sim 650 \mathrm{~nm}$ 处接收.

\section{5 细胞毒性实验}

细胞毒性实验用 MTT 方法得到. 细胞首先被培养 在 96 孔板中, 每孔 $6 \times 10^{3}$ 个, 培养过夜. 之后加入不同 浓度和种类的材料及药物, 在 $24 / 48 \mathrm{~h}$ 后, MTT 活性物(5 $\mathrm{mg} / \mathrm{mL}, 20 \mu \mathrm{L}$ )被加入到细胞中从而生成甲㬺，继续在 $37{ }^{\circ} \mathrm{C}$ 培养 $4 \mathrm{~h}$, 用二甲基亚砜(DMSO)溶解甲肤，并用 酶标仪(SUNOSTIK SPR-960)在 $490 \mathrm{~nm}$ 波长处进行定量 表征.

\section{References}

[1] Morelli, D.; Menard, S.; Colnaghi, M. I.; Balsari, A. Cancer Res. 1996, 56, 2082

[2] (a) Vonhoff, D.; Layard, M. W.; Basa, P.; Davis, H. L.; Vonhoff, A. L.; Rozencweig, M.; Muggia, F. M. Ann. Intern. Med. 1979, 91, 710; (b) Byrne, J. D.; Betancourt, T.; Brannon-Peppas, L. Adv. Drug Delivery Rev. 2008, 60, 1615.

[3] Gabizon, A.; Shmeeda, H.; Barenholz, Y. Clin. Pharmacokinet. 2003, 42, 419.

[4] (a) Liu, Z.; Fan, A. C.; Rakhra, K.; Sherlock, S.; Goodwin, A.; Chen, X. Y.; Yang, Q. Y.; Felsher, D. W.; Dai, H. J. Angew. Chem., Int. Ed. 2009, 48, 7668; (b) Liu, Z.; Sun, X. M.; NakayamaRatchford, N.; Dai, H. J. ACS Nano 2007, 1, 50.

[5] (a) Vivero-Escoto, J. L.; Slowing, I. I.; Trewyn, B. G.; Lin, V. S. Y. Small 2010, 6, 1952; (b) Yuan, L.; Wang, B. D.; Tang, Q. Q.; Zhang, X. H.; Yang, D.; Hu, J. H. Chin. J. Org. Chem. 2010, 30 640. (袁丽, 王蓓娣, 唐倩倩, 张晓鸿, 张晓环, 杨东, 胡建华, 有 机化学, 2010, 30, 640.)

[6] (a) Kim, T. W.; Chung, P. W.; Slowing, I. I.; Tsunoda, M.; Yeung, E. S.; Lin, V. S. Y. Nano Lett. 2008, 8, 3724; (b) Gu, J. L.; Su, S. S.; Li, Y. S.; He, Q. J.; Shi, J. L. Chem. Commun. 2011, 47, 2101.

[7] Nie, S. M.; Xing, Y.; Kim, G. J.; Simons, J. W. Annu. Rev. Biomed. Eng. 2007, 9, 257.

[8] Zhu, J.; Liao, L.; Bian, X. J.; Kong, J. L.; Yang, P. Y.; Liu, B. H. Small 2012, 8, 2715.

[9] Fang, Y.; Gu, D.; Zou, Y.; Wu, Z. X.; Li, F. Y.; Che, R. C.; Deng, Y. H.; Tu, B.; Zhao, D. Y. Angew. Chem., Int. Ed. 2010, 49, 7987.

[10] Zhang, X. K.; Meng, L. J.; Lu, Q. H.; Fei, Z. F.; Dyson, P. J. Biomaterials 2009, 30, 6041.

[11] Xiong, L. Q.; Chen, Z. G.; Yu, M. X.; Li, F. Y.; Liu, C.; Huang, C. H. Biomaterials 2009, 30, 5592

[12] Liu, J. H.; Cao, L.; Luo, P. J. G.; Yang, S. T.; Lu, F. S.; Wang, H. F.; Meziani, M. J.; Haque, S. A.; Liu, Y. F.; Lacher, S.; Sun, Y. P. ACS Appl. Mater. Interfaces 2010, 2, 1384. 